\title{
The exact analytical solution of the linear structure growth rate in $\Lambda$ CDM cosmology and its cosmological applications
}

\author{
Pengjie Zhang1,* \\ ${ }^{1}$ Key laboratory for research in galaxies and cosmology, Shanghai Astronomical Observatory, \\ Chinese Academy of Science, 80 Nandan Road, Shanghai, China, 200030
}

\begin{abstract}
We derive the exact analytical solution of the linear structure growth rate in $\Lambda$ CDM cosmology with flat or curved geometry, under the Newtonian gauge. Unlike the well known solution under the Newtonian limit (Heath 1977, [1] ), our solution takes all general relativistic corrections into account and is hence valid at both the sub- and super-horizon scales. With this exact solution, we evaluate cosmological impacts induced by these relativistic corrections. (1) General relativistic corrections alter the density growth from $z=100$ to $z=0$ by $10 \%$ at $k=0.01 \mathrm{~h} / \mathrm{Mpc}$ and the impact becomes stronger toward larger scales. We caution the readers that the overdensity is not gauge invariant and the above statement is restrained to the Newtonian gauge. (2) Relativistic corrections introduce a $k^{-2}$ scale dependence in the density fluctuation. It mimics a primordial non-Gaussianity of the local type with $f_{\mathrm{NL}}^{\text {local }} \sim 1$. This systematical error may become non-negligible for future all sky deep galaxy surveys. (3) Cosmological simulations with box size greater than $1 \mathrm{Gpc}$ are also affected by these relativistic corrections. We provide a post-processing recipe to correct for these effects. (4) These relativistic corrections affect the redshift distortion. However, at redshifts and scales relevant to redshift distortion measurements, such effect is negligible.
\end{abstract}

PACS numbers: 98.65.Dx; 04.25.Nx

\section{INTRODUCTION}

A well known and widely used solution of the linear density growth in $\Lambda \mathrm{CDM}$ cosmology, first derived by Heath 1977 [1], is

$$
\delta_{m} \propto D_{m, N} \propto H \int_{0}^{a} \frac{d a}{H^{3} a^{3}} .
$$

Here, $\delta_{m}$ is the matter overdensity. $D_{m, N}$ is the linear density growth rate in the Newtonian limit. Throughout the paper, the subscript " $\mathrm{N}$ " denotes the corresponding property in the Newtonian limit. $H=H(a)$ is the Hubble parameter at redshift $z=1 / a-1$ and $a$ is the scale factor. This solution is derived under the limit of negligible radiation, negligible baryon pressure and identical initial conditions for fluctuations in baryons and dark matter. These conditions are adopted throughout the paper.

This solution is valid for arbitrary cosmological constant $\Lambda$. It is also valid for arbitrary curvature $K$, at scales much smaller than the curvature radius $r_{K}=$ $1 / \sqrt{K}=1 / H_{0} \sqrt{\left|\Omega_{K}\right|}$. Here $\Omega_{K}$ is the dimensionless curvature density and $H_{0} \equiv H(a=1)$ is the present day Hubble constant. Throughout the paper, we set the speed of light $c=1$, so $1 / H_{0}=3 h^{-1} \mathrm{Gpc}$.

However, this solution is derived by neglecting relativistic corrections to the Poisson equation and to the continuity equation, so it is valid only in the Newtonian limit and hence at sub-horizon scale, where the wavevec-

\footnotetext{
*Electronic address: pjzhang@shao.ac.cn
}

tor $k$ satisfies

$$
k \gg a H=\frac{a \sqrt{\Omega_{0} a^{-3}+\Omega_{\Lambda}+\Omega_{K} a^{-2}}}{3 \times 10^{3} h^{-1} \mathrm{Mpc}} .
$$

Here, $\Omega_{0}$ and $\Omega_{\Lambda}$ are the dimensionless matter and $\Lambda$ today, respectively.

Modern surveys are pushing the observational boundary to larger scale and higher redshift. This enables several important cosmological applications. (1) Galaxy clustering at scales $k \lesssim 0.01 \mathrm{~h} / \mathrm{Mpc}$ is a sensitive probe of primordial non-Gaussianity (PMG). It relies on the asymptotic behavior of galaxy clustering at $k \rightarrow 0$ to probe PMG (e.g. 2]). (2) The large scale structure (LSS) growth history, often inferred by combining low redshift and high redshift measurements, contains key information to test general relativity and probe dark energy. Such measurement is usually done at $k \gtrsim 0.01 h / \mathrm{Mpc}$ scale. However, since the horizon scale decreases with increasing redshift, even a mode deep in the sub-horizon regime today can be close to the horizon scale at earlier epoch.

For these cosmological applications, whether relativistic corrections can be safely neglected and whether Eq. 1 is sufficiently accurate are becoming issues of practical importance and have evoked discussions (e.g. [3]). Existing packages such as CMBFAST [4] and CAMB [5] numerically solve the fully general relativistic linear perturbation equations. So they can be reliably resorted to calculate the structure growth at all linear scales.

However, it is still useful to understand the role of these relativistic corrections through analytical approach. This motivates us to derive the exact solution of the linear density evolution with all relativistic corrections included, 
valid at both the sub-horizon and super-horizon, for arbitrary $\Lambda$ and $K$.

For LSS study, the most widely used gauge is the Newtonian gauge (or the conformal Newtonian gauge). Hence we will perform the derivation of the exact solution in the Newtonian gauge, and then discuss its implications on the LSS-based cosmology. Some of the perturbation variables in the Newtonian gauge, such as the matter overdensity $\delta_{m}$, are not gauge invariant. However, such gauge freedom is not a problem, as long as we properly connect the perturbation variables in the Newtonian gauge to observables (refer to [6] for a comprehensive treatment). Furthermore, once the solution in the Newtonian gauge is derived, it can be transformed to other gauges by performing a coordinate transformation (e.g. [7, 8]). For these reasons, we will stick to the Newtonian gauge throughout the paper.

After we derived the solution and were writing this manuscript, we found an earlier work by Jai-Chan Hwang [9], who has derived equivalent solutions in not only the Newtonian gauge, but also in other five gauges. Nevertheless, we feel the need to publish our result, for a number of reasons. First, the solutions are derived in independent ways. [9] first derived the solution in the comoving gauge and then converted to the Newtonian gauge (denoted as the zero shear gauge in [9]). For us, we derive the solution directly in the Newtonian gauge. Our result thus serves as an independent check and the agreement between the two verifies the validity of both solutions. Second, given recent discussions on the role of these relativistic corrections (e.g. [3]), it is useful to address the existence of these exact solutions and clarify some confusions arisen in the literature. Third, the exact solution derived has direct applications to modern cosmological topics, some of them, e.g. the primordial non-Gaussianity, will be elaborated later in the paper.

The exact analytical solution that we found is valid for any $\Lambda$ and curvature $K$. But numerical results and related discussions are based on the standard flat $\Lambda$ CDM with $\Omega_{0}=0.268, \Omega_{\Lambda}=1-\Omega_{0}$ and $\Omega_{K}=0$ throughout the paper, unless otherwise specified.

\section{THE EXACT SOLUTION}

We work with the FRW background metric

$$
\begin{aligned}
d s^{2} & \equiv-g_{\mu \nu} d x^{\mu} d x^{\nu}=-d t^{2}+a^{2} \gamma_{i j} d x^{i} d x^{j} \\
& =-d t^{2}+a^{2}\left(\frac{d r^{2}}{1-K r^{2}}+r^{2} d \theta^{2}+r^{2} \sin ^{2} \theta d \varphi^{2}\right) .
\end{aligned}
$$

The Hubble expansion rate is given by

$$
H^{2}=\frac{8 \pi G}{3}\left(\bar{\rho}_{m}+\rho_{\Lambda}\right)-\frac{K}{a^{2}} .
$$

Here $\bar{\rho}_{m}$ is the mean cosmological matter density. Taking the derivative of both sides, we obtain a relation which will be used later,

$$
K=4 \pi G \bar{\rho}_{m} a^{2}+H H^{\prime} a^{3} .
$$

Throughout the paper, ${ }^{\prime} \equiv d / d a$.

For reviews of linear perturbation in $\Lambda \mathrm{CDM}$ cosmology, please refer to, e.g, [7, 8, 10]. Here we just summarize relevant results. For the convenience of LSS study, we adopt the Newtonian gauge defined through

$$
d s^{2}=-(1+2 \phi) d t^{2}+a^{2}(1-2 \phi) \gamma_{i j} d x^{i} d x^{j} .
$$

Here, we have adopted the result that the two Newtonian potentials are identical in $\Lambda \mathrm{CDM}$ (e.g. 7]). We choose the following three independent perturbation equations,

$$
\begin{gathered}
\left(\nabla^{2}+3 K\right) \phi-3 a^{2} H^{2}\left(\phi^{\prime} a+\phi\right)=4 \pi G \bar{\rho}_{m} a^{2} \delta_{m}, \\
a H\left(\phi^{\prime} a+\phi\right)=4 \pi G \bar{\rho}_{m} a^{2} W \\
\delta_{m}^{\prime}=\frac{\nabla^{2} W}{a^{2} H}+3 \phi^{\prime} .
\end{gathered}
$$

Here, $\nabla^{2} \equiv \gamma^{i j} \nabla_{; i} \nabla_{; j}$ and the covariant derivative $\nabla_{; i}$ is defined in the $3 \mathrm{D}$ constant curvature space. $\vec{v}=-\nabla W$ is the peculiar velocity and $W$ is the velocity potential. The three equations above compose a complete set for the evolution of the three perturbation variables, the Newtonian potential $\phi$, the matter overdensity $\delta_{m}$ and the velocity potential $W$.

The $\phi^{\prime} a+\phi$ term in Eq. 7 is a relativistic correction to the Poisson equation. The $\phi^{\prime}$ term in Eq. 9 reflect the ambiguity to distinguish inhomogeneities in spacetime metric to that in the matter-energy fluid. These relativistic corrections are usually neglected in LSS study. It is the goal of this paper to quantify their impacts.

\section{Linear evolution of the gravitational potential and peculiar velocity}

Interestingly, including all these relativistic terms allows us to derive the exact solution. From Eq. 7, we obtain $\delta_{m}^{\prime}$,

$$
\delta_{m}^{\prime}=\left[\frac{\left(\nabla^{2}+3 K\right) \phi-3 a^{2} H^{2}\left(\phi^{\prime} a+\phi\right)}{4 \pi G \bar{\rho}_{m} a^{2}}\right]^{\prime} .
$$

Plug the above equation and Eq. 8 into Eq. 9, we obtain

$$
\left[\frac{\left(\nabla^{2}+3 K\right) \phi-3 a^{2} H^{2}\left(\phi^{\prime} a+\phi\right)}{4 \pi G \bar{\rho}_{m} a^{2}}\right]^{\prime}=\frac{\nabla^{2}\left(\phi^{\prime} a+\phi\right)}{4 \pi G \bar{\rho}_{m} a^{3}}+3 \phi^{\prime}
$$

Since $\bar{\rho}_{m} \propto a^{-3}$, in the above equation, the two terms $\propto \nabla^{2}$ cancel exactly. We then have

$$
\left[\frac{3 K \phi-3 a^{2} H^{2}\left(\phi^{\prime} a+\phi\right)}{4 \pi G \bar{\rho}_{m} a^{2}}\right]^{\prime}=3 \phi^{\prime}
$$


The solution is

$$
\frac{a^{2} H^{2}\left(\phi^{\prime} a+\phi\right)-K \phi}{4 \pi G \bar{\rho}_{m} a^{2}}+\phi=\text { constant } .
$$

Immediately we see the linear evolution in $\phi$ is scale independent. This tells us that the solution of $\phi$ obtained in the sub-horizon limit is also valid at super-horizon scale.

Multiple both sides by $4 \pi G \bar{\rho}_{m} a^{3} / H^{3} a^{3}$ and use Eq. 囵, we obtain

$$
\left(\frac{a \phi}{H}\right)^{\prime} \propto \frac{1}{H^{3} a^{3}}
$$

Here, we have used the fact that $4 \pi G \bar{\rho}_{m} a^{3}$ is a constant.

The above equation has two independent solutions. The solution $\phi \propto H / a$ corresponds to the decay mode in density. So it is of little practical interest and will not be discussed hereafter. The other solution corresponds to the growth mode in the density evolution,

$$
\phi \propto D_{\phi} \propto \frac{H}{a} \int_{0}^{a} \frac{d a}{H^{3} a^{3}} .
$$

Now the scale independence in $D_{\phi}$ is explicitly shown.

The linear evolution in velocity (divergence) can be obtained combining Eq. 8 \& 15 ,

$$
W \propto D_{v} \propto a H^{2}\left(\frac{H^{\prime} a}{H}+\frac{1 / H^{3} a^{2}}{\int_{0}^{a} d a / H^{3} a^{3}}\right) \int_{0}^{a} \frac{d a}{H^{3} a^{3}} .
$$

Here,

$$
\frac{H^{\prime} a}{H}+\frac{1 / H^{3} a^{2}}{\int_{0}^{a} d a / H^{3} a^{3}}=\frac{d \ln \phi}{d \ln a}+1 .
$$

For the standard $\Lambda \mathrm{CDM}$, this quantity approaches unity at high redshift and decreases to $\sim 0.5$ at $z=0$.

We notice that both the evolution in the gravitational potential and in the velocity are identical to the ones derived in the sub-horizon (Newtonian) limit, namely,

$$
D_{\phi}=D_{\phi, N} \quad ; \quad D_{v}=D_{v, N}
$$

Indeed, this is what expected from the scale independence in $D_{\phi}$ and $D_{v}$.

\section{Linear evolution of the matter overdensity}

However, the situation for the overdensity evolution is different. From Eq. 7

$$
\begin{aligned}
\delta_{m}= & \frac{2 \Omega_{0} a}{3 H_{0}^{2}} \times \\
& {\left[\nabla^{2}+3 K-3 a^{2} H^{2}\left(\frac{H^{\prime} a}{H}+\frac{1 / H^{3} a^{2}}{\int_{0}^{a} d a / H^{3} a^{3}}\right)\right] \phi . }
\end{aligned}
$$

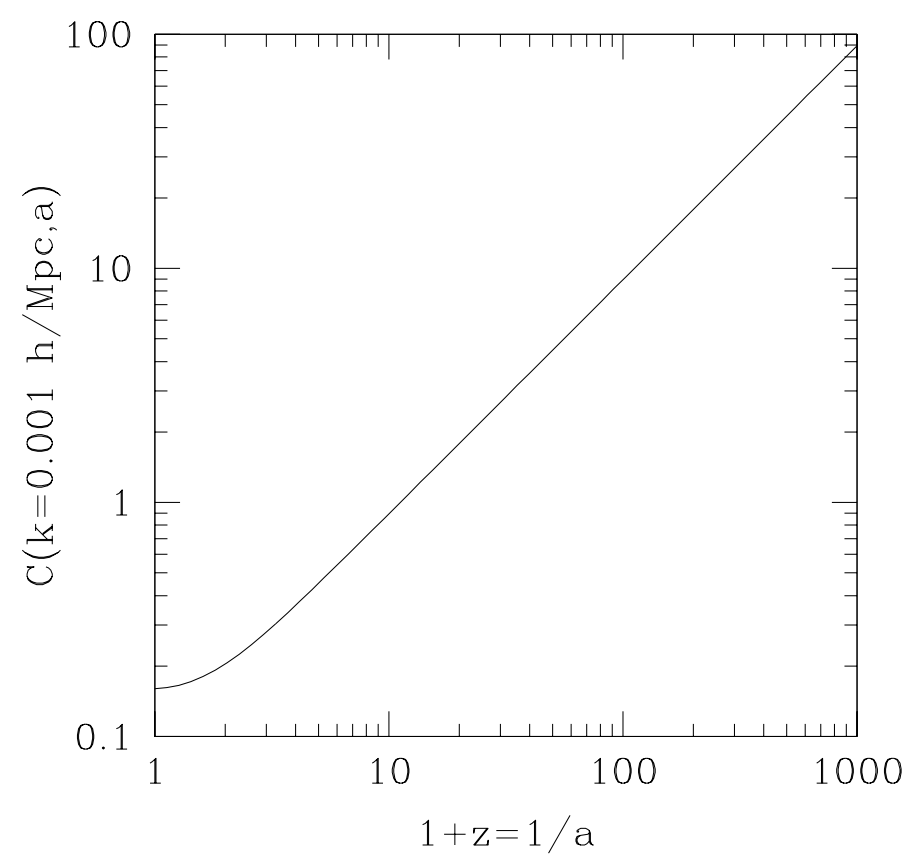

FIG. 1: The relativistic correction $C(k, a)$. Relativistic corrections alter the matter power spectrum by a factor $(1+C)^{2}$. So a positive $C$ means the amplification of the matter clustering. Since $C \propto k^{-2}$, we only plot $C(k, a)$ at $k=0.001 h / \mathrm{Mpc}$. At $z \gg 1, C(k, a) \propto a^{2}$ since $d \ln \phi / d \ln a \rightarrow 0$.

Only when the scale is much smaller than the curvature radius and the horizon scale, we recover Eq. 1]

CMB observations, along with other probes, show the universe to be nearly flat $\left(\left|\Omega_{K}\right| \ll 1\right)[1]$. Hence all modes accessible to observations are much smaller than the curvature radius. We then neglect the curvature term and replace $\nabla^{2}$ with the usual Laplace operator in the 3D Euclid space. We further proceed to the Fourier space $\left(\nabla^{2} \rightarrow-k^{2}\right)$. We denote the corresponding Fourier components with a superscript " $\sim$ ". We then have

$$
\tilde{\delta}_{m}=-\frac{2 \Omega_{0} a}{3 H_{0}^{2}} k^{2} \tilde{\phi} \times[1+C(k, a)] .
$$

The impact of relativistic corrections is then completely captured by the relativistic correction term

$$
\begin{aligned}
C(k, a) & =\frac{3 a^{2} H^{2}}{k^{2}}\left(\frac{H^{\prime} a}{H}+\frac{1 / H^{3} a^{2}}{\int_{0}^{a} d a / H^{3} a^{3}}\right) \\
& =\frac{a^{2}\left(H / H_{0}\right)^{2}}{3\left(k \times 10^{3} h^{-1} \mathrm{Mpc}\right)^{2}}\left(\frac{H^{\prime} a}{H}+\frac{1 / H^{3} a^{2}}{\int_{0}^{a} d a / H^{3} a^{3}}\right) .
\end{aligned}
$$

For the standard $\Lambda \mathrm{CDM}$ cosmology, $C>0$. The numerical result is plotted in Fig. 1 .

The matter power spectrum $P_{m}$ is related to the power spectrum of the gravitational potential $P_{\phi}$ through Eq. 


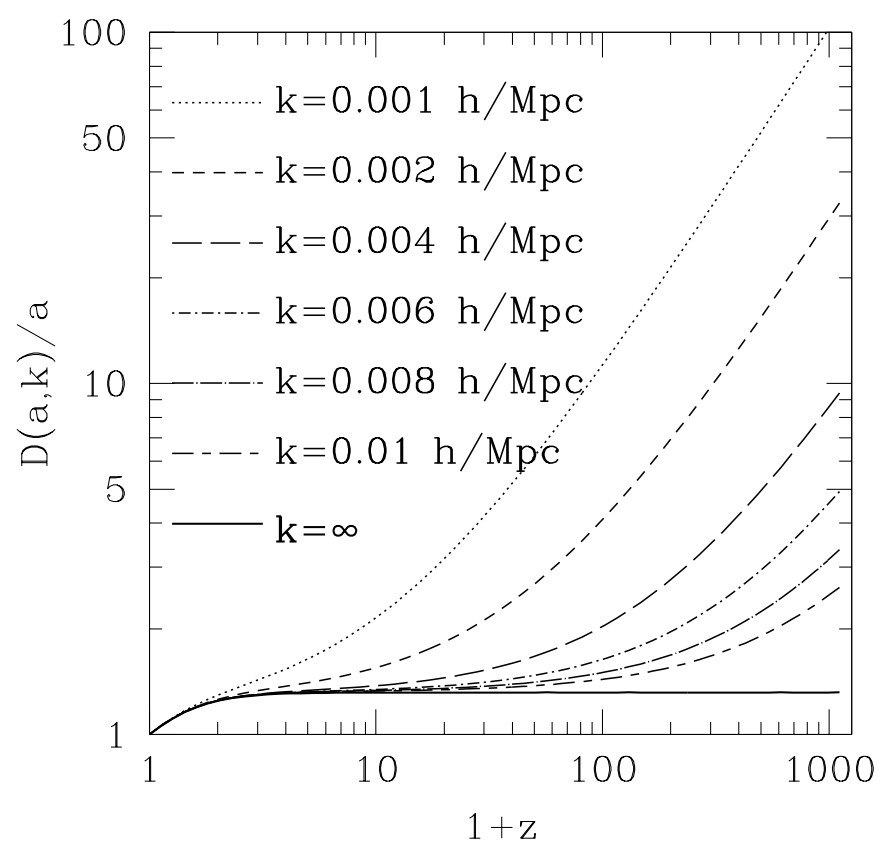

FIG. 2: The linear density evolution in $\Lambda$ CDM under the Newtonian gauge, for various $k$. The solid line corresponds to the one in the Newtonian limit. Larger deviation from the Newtonian result happen at higher redshifts, where the relativistic corrections are larger due to smaller horizon scale.

20,

$$
\begin{aligned}
P_{m}(k, z) & =\left[\frac{2 \Omega_{0} a}{3 H_{0}^{2}}\right]^{2} k^{4} P_{\phi} \times[1+C(k, a)]^{2} \\
& =P_{m, N}(k, z) \times[1+C(k, a)]^{2} .
\end{aligned}
$$

Since $C>0$, relativistic corrections enhance the matter clustering.

The linear density evolution is derived combining Eq. 20] \& 15.

$$
\tilde{\delta}_{m} \propto\left[H \int_{0}^{a} \frac{d a}{H^{3} a^{3}}\right] \times[1+C(k, a)] .
$$

Relativistic corrections induce a characteristic scale dependence $k^{-2}$ in the otherwise scale independent density growth in $\Lambda$ CDM cosmology. Only when $k \gg a H$, does this scale dependence vanishes $(C(k, a) \ll 1)$. The linear density growth factor $D_{m}$ is often defined as $D_{m} \equiv$ $\delta_{m}(a) / \delta_{m}(a=1)$ and normalized at $z=0$, in LSS study. We then have

$$
\begin{aligned}
D_{m}(a, k) & =\left[\frac{H(a)}{H_{0}}\right]\left[\frac{\int_{0}^{a} d a / H^{3} a^{3}}{\int_{0}^{1} d a / H^{3} a^{3}}\right]\left[\frac{1+C(k, a)}{1+C(k, a=1)}\right] \\
& =D_{m, N}(a) \times\left[\frac{1+C(k, a)}{1+C(k, a=1)}\right] .
\end{aligned}
$$

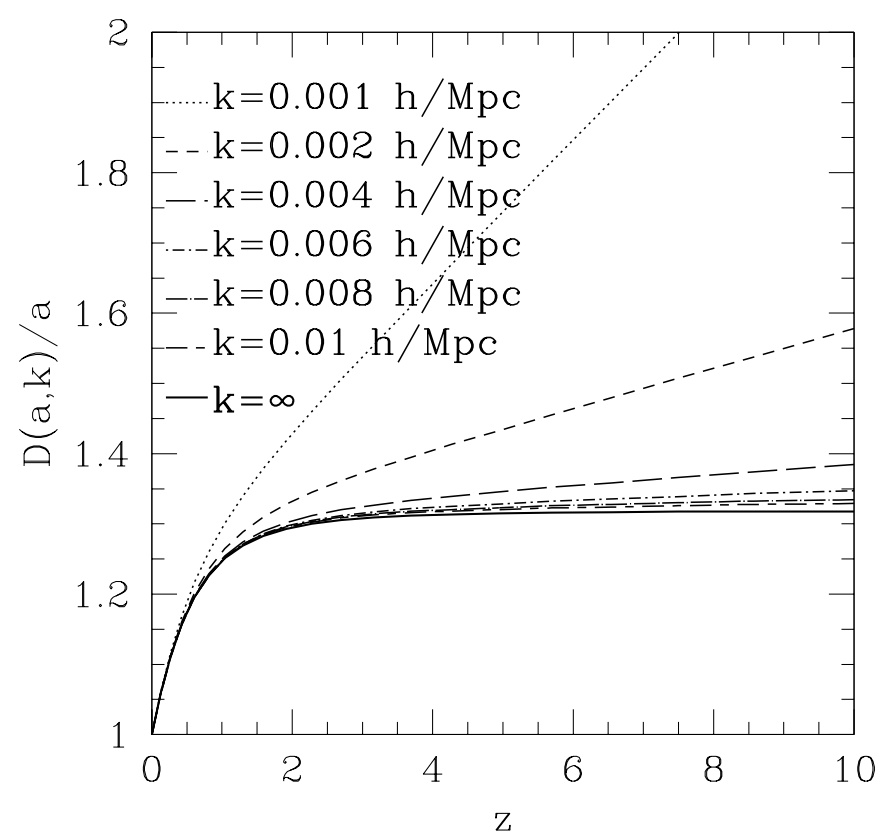

FIG. 3: Same at Fig. 2, but only show the evolution at $z<10$ where LSS surveys may have access.

We plot $D(a, k) / a$ for various $k$ in Fig. 2 \& 3 . As expected, the relativistic corrections become bigger at higher redshifts and can completely invalidate the Newtonian result (Eq. 1), due to shrinking horizon. This analytical result verifies the finding of [3], who used numerical calculation to quantify the relativistic corrections. One thing to notice is that, 3. normalizes the overdensity at $z=1100$ and hence defines $D=\delta_{m}(z) / \delta_{m}(z=1100)$, so deviations from the Newtonian limit appear at lower redshift instead.

Surprisingly, the large deviation shown in Eq. 11 and Fig. 2 does not necessarily invalidate the applicability of the Newtonian approximation (Eq. 1) in LSS study. This is essentially a normalization issue. To better demonstrate this point, we highlight the linear density growth at $z<10$ in Fig. 3. Most LSS surveys are limited to $z \lesssim 4$. If we solely compare LSS at redshifts accessible to those surveys to measure the structure growth rate, Eq. 1 is essentially exact for $k>0.01 h / \mathrm{Mpc}$. Even for the mode $k=0.006 h / \mathrm{Mpc}$, the accuracy is better than $1 \%$, negligible comparing to the cosmic variance. Hence in general, Eq. 1 is sufficiently accurate to describe the linear growth at redshifts accessible to LSS surveys at $z \lesssim 4$. However, later in the paper we will discuss an important exception, where by the multiple tracer technique proposed by [12], the cosmic variance can be eliminated.

$21 \mathrm{~cm}$ surveys can probe LSS to the reionization epoch at $z \sim 10$ and even higher 13. A comparison between these epochs and $z \sim 0$ based on the Newtonian approximation (Eq. 1) would lead to $\gtrsim 1 \%$ error in the derived 


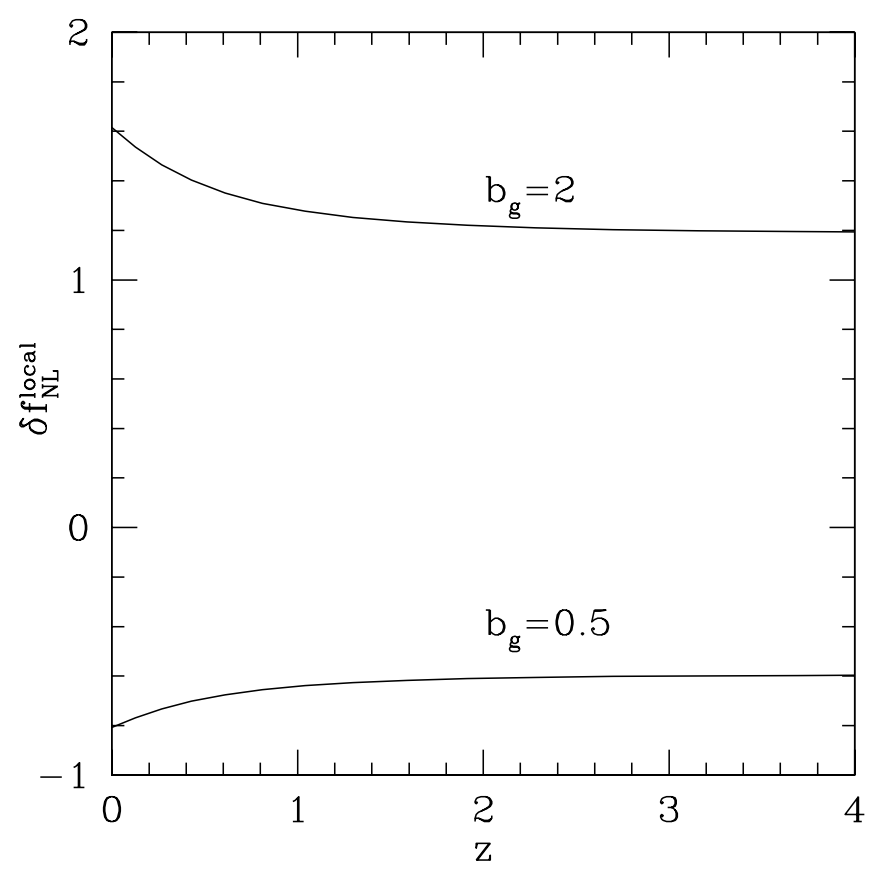

FIG. 4: General relativistic corrections to the Poisson equation and the continuity equation can mimic a primordial non-Gaussianity of local type and induce a bias $\delta f_{\mathrm{NL}}^{\text {local }} \propto$ $b_{g} /\left(b_{g}-1\right)$. The plot shows the case for $b_{g}=2$ and $b_{g}=0.5$ respectively.

density growth rate at $k=0.01 \mathrm{~h} / \mathrm{Mpc}$, still small, but may no longer be negligible.

On the other hand, if we want to compare LSS measurements at low redshift to $\mathrm{CMB}$ at $z \simeq 1100$, Eq. 1 is no longer applicable, shown in Fig. 2, Even for $k=0.01 \mathrm{~h} / \mathrm{Mpc}$, the error induced is a factor of 2 . Nevertheless, there are other sources of error prohibiting the application of Eq. 1 in such case, such as non-negligible radiation and non-identical baryon and dark matter distribution at $z \gtrsim 100$. CMB packages such as CMBFAST and CAMB have already taken these complexities into account and shall be used to compare between high and low redshift observations.

\section{COSMOLOGICAL IMPACTS OF RELATIVISTIC CORRECTIONS}

In this section we discuss the impact of these relativistic corrections on a number of cosmological applications of LSS. We find that non-negligible impact may occur for searching for primordial non-Gaussianity through twopoint galaxy clustering and N-body simulations of Gpc box size.

\section{Primordial non-Gaussianity}

The galaxy overdensity $\delta_{g}$ is an observable and hence gauge independent. In the Newtonian gauge, it can be expressed as [6]

$$
\delta_{g}=b_{g} \delta_{m}+f(\phi, \vec{v}) .
$$

Here, $f$ is a linear function of $\phi$ and $\vec{v}$. We have shown that the relativistic corrections discussed earlier do not bias the Newtonian calculation on $\phi$ and $\vec{v}$, so the only term affected by the relativistic corrections is the usual $b_{g} \delta_{m}$ term, where $b_{g}$ is the galaxy bias.

Primordial non-Gaussianity (PMG) of the local type induces scale dependence to the otherwise scaleindependent bias at large scale [2],

$$
\Delta b_{g}=2\left(b_{g}-1\right) f_{\mathrm{NL}}^{\text {local }} \delta_{c} \frac{3 \Omega_{m} H_{0}^{2}}{2 a g(a) k^{2}} .
$$

Here, $f_{\mathrm{NL}}^{\text {local }}$ is the PMG parameter. $\delta_{c}$ is the threshold for halo collapse and $g \propto D(k \rightarrow \infty) / a$, normalized such that $g(a \rightarrow 0) \rightarrow 1$. If we miss the relativistic corrections in the matter evolution, it will bias the interpretation of galaxy bias by

$$
\delta b_{g}=b_{g} C(k, a) .
$$

This in turn causes a systematical error in $f_{\mathrm{NL}}^{\text {local }}$,

$$
\begin{aligned}
\delta f_{\mathrm{NL}}^{\text {local }} & =\frac{b_{g} a^{3} H^{2} g(a)}{\left(b_{g}-1\right) \delta_{c} \Omega_{0} H_{0}^{2}}\left(\frac{H^{\prime} a}{H}+\frac{1 / H^{3} a^{2}}{\int_{0}^{a} d a / H^{3} a^{3}}\right) \\
& =\frac{b_{g}}{b_{g}-1} \times O(1) .
\end{aligned}
$$

It diverges when $b_{g} \rightarrow 1$. But this divergence is trivial, simply meaning that PMG of the local type does not affect the two-point clustering of $b_{q}=1$ galaxies (Eq. $[26$ and more detailed discussions in [2]). The induced biases for $b_{g}=2$ and $b_{g}=0.5$ are shown in Fig. 4. Existing constraints on $f_{\mathrm{NL}}^{\text {local }}$ from two-point galaxy clustering [14] are at least one order of magnitude larger, so the bias induced by relativistic corrections is negligible for existing surveys and stage IV surveys such as LAMOST [15] and BOSS. However, deep full sky LSS surveys have the potential to constrain $f_{\mathrm{NL}}^{\text {local }}$ with statistical error $\Delta f_{\mathrm{NL}}^{\text {local }}=O(1)[12]$. For these surveys, the relativistic correction induced bias should be included in the analysis.

Terms linear in $\phi$ in Eq. 25] also induce bias to $f_{\mathrm{NL}}^{\text {local }}[\underline{6}$, 16]. The two systematical biases in $f_{\mathrm{NL}}^{\text {local }}$ are independent and comparable in amplitude.

\section{Large box N-body simulation}

Large simulation box size $L \sim 1 h^{-1} \mathrm{Gpc}$ is required to calculate the nonlinear matter power spectrum at $1 \%$ 
level accuracy [17]. This is also the requirement for primordial non-Gaussianity study. N-body simulation usually adopts the Poisson equation $\nabla^{2} \phi=4 \pi G \bar{\rho}_{m} a^{2} \delta_{m}$ to calculate the potential and hence misses relativistic corrections. The largest modes accessible to these simulations are $k \sim 2 \pi / L \sim 0.006 h / \mathrm{Mpc}$. N-body simulations typically begin at $z=100$. For the largest modes, the density evolution to $z=0$ is then biased by more than $20 \%$, definitely non-negligible.

This problem can be solved in the post-processing of simulation. Eq. 18 means that the simulated gravitational potential $\phi$ is correct. So from Eq. 7 by simply adding a term $-2 a^{3}\left(H / H_{0}\right)^{2}\left(\phi^{\prime} a+\phi\right) / \Omega_{0}$ to the simulated $\delta_{m}$, we can recover the correct density evolution.

\section{Redshift distortion}

Redshift distortion is emerging as a promising probe of the large scale structure [18]. Cosmological information encoded in redshift distortion is often quantified by a single parameter $f$. The usual definition is $f \equiv d \ln D_{m} / d \ln a$. Through this definition, one can work out the impact of relativistic corrections straightforwardly.

Alternatively, one can define it as

$$
f \equiv \frac{-\theta / a H}{\delta_{m}} \equiv \frac{\nabla^{2} W / a H}{\delta_{m}} .
$$

This definition does not assume a deterministic peculiar velocity-overdensity relation and is hence more general. We then have

$$
f=\frac{H^{\prime} a / H+1 / H^{3} a^{2} \int_{0}^{a} d a / H^{3} a^{3}}{1+C(k, a)}=\frac{f_{N}}{1+C(k, a)} .
$$

Here, $f_{N}$ is the $f$ in the Newtonian limit. Relativistic corrections induce scale dependence in $f$, quantified by $C(k, a)$.

The $\gamma$-index is often adopted to describe the structure growth [19]. If we straightforwardly extend the usual definition, we have

$$
\begin{aligned}
\gamma(a, k) & =\frac{\ln f}{\ln \Omega_{m}(a)} \\
& =\gamma_{N}(a)-\frac{\ln [1+C(k, a)]}{\ln \Omega_{m}(a)} \simeq \gamma_{N}-\frac{C(k, a)}{\ln \Omega_{m}(a)} .
\end{aligned}
$$

For most scales and redshifts accessible to the precision redshift distortion measurement, $C \sim(k \times$ $\left.10^{3} h^{-1} \mathrm{Mpc}\right)^{-2} \ll 1$. So relativistic corrections have negligible impact on $f$ and $\gamma$.

\section{SUMMARY}

We consider relativistic corrections to the Poisson equation and the continuity equation and investigate their impact to the linear evolution of perturbations in $\Lambda$ CDM. Major results are as follows.

- We derive the exact analytical solution for the linear evolution of gravitational potential, velocity and matter overdensity under the Newtonian gauge. It is valid at both the sub- and superhorizon scale, for arbitrary cosmological constant and curvature.

- Relativistic corrections amplify the matter clustering and introduce a $k^{-2}$ scale dependence to the density fluctuation. The overdensity evolution is altered by $10 \%$ at $k=0.01 \mathrm{~h} / \mathrm{Mpc}$ from $z=100$ to $z=0$.

- The gravitational potential and velocity evolution obtained under the Newtonian limit remaines exact with the presence of these relativistic corrections.

- If these relativistic corrections are not included in analyzing the two-point galaxy clustering, constraint on the primordial non-Gaussianity will be biased by $\delta f_{\mathrm{NL}}^{\text {local }} \sim 1$.

- These relativistic corrections bias N-body simulations, which are based on the Newtonian approximation. For $\sim h^{-1} \mathrm{Gpc}$ box size simulations, the largest mode of density fluctuations is underestimated by $\sim 20 \%$ from $z=100$ to $z=0$. We further show that this effect is correctable in the simulation post-processing.

One question remains is whether we can extend the analytical solution to dark energy models. Under the limit that dark energy fluctuation is negligible, Eq. 7, 8 \& 9 hold. So it seems that we can repeat the derivation for smooth dark energy models up to Eq. 13. which allows us to express the linear evolution with a double integral form. Unfortunately, numerically we find that the solution obtained in this way does not reproduce the known (and correct) behavior at deep sub-horizon scale. The reason is that, dark energy fluctuations are inevitable since $w \neq-1$ [20]. It is true that these fluctuations can be small and negligible with respect to the dominant terms in Eq. 7, 8\& 9. However, they are comparable to $\phi$ in Eq. 13, By neglecting them at first hand in Eq. 7,8 \& 9. we miss non-negligible corrections from dark energy fluctuations to Eq. 13 .

\section{ACKNOWLEDGMENT}

I thank Jun Zhang and Xuelei Chen for many helpful discussions. This work is supported by the onehundred talents program of the Chinese academy of science, the national science foundation of China (grant No. 
10821302, 10973027\& 11025316), the CAS/SAFEA International Partnership Program for Creative Research Teams and National Basic Research Program of China (973 Program) under grant No.2009CB24901.

[1] D.J. Heath, MNRAS, 179, 351 (1977)

[2] Neal Dalal, Olivier Dor, Dragan Huterer, Alexander Shirokov. Phys.Rev.D77:123514 (2008). arXiv:0710.4560

[3] James B. Dent, Sourish Dutta. Phys. Rev. D 79, 063516 (2009). arXiv:0808.2689

[4] Uros Seljak, Matias Zaldarriaga. ApJ, 469, 437 (1996)

[5] A. Lewis, A. Challinor. http://camb.info/

[6] Jaiyul Yoo, A. Liam Fitzpatrick, Matias Zaldarriaga. Phys.Rev.D80:083514 (2009). arXiv:0907.0707

[7] Chung-Pei Ma, Edmund Bertschinger. Astrophys.J. 455 (1995) 7-25

[8] Kodama, Hideo; Sasaki, Misao. Progress of Theoretical Physics Supplement, Vol. 78, p. 1, 1984

[9] Hwang, J.-C. 1994, Astrophys. J. , 427, 533

[10] Edmund Bertschinger. arXiv:astro-ph/0101009

[11] P. de Bernardis, et al. Nature, 404, 955 (2000); E. Komatsu, et al. arXiv:1001.4538 (2010)

[12] Uros Seljak. Phys.Rev.Lett.102:021302 (2009)

[13] e.g. Abraham Loeb, Matias Zaldarriaga. PRL, 92, 211301 (2004); U. Pen, New Astron. 9, 417 (2004); Xuelei Chen, Jordi Miralda-Escude. ApJ, 684,18 (2008) arXiv:astro-ph/0605439 Pengjie Zhang, Zheng Zheng, Renyue Cen. MNRAS, 382, 1087 (2007)

[14] Anze Slosar, Christopher Hirata, Uros Seljak, Shirley Ho, Nikhil Padmanabhan. JCAP 08, 031 (2008)

[15] Yan Gong, Xin Wang, Zheng Zheng, Xuelei Chen. Res.Astron.Astrophys.10, 107 (2010)

[16] Jaiyul Yoo. arXiv:1009.3021 (2010)

[17] Katrin Heitmann, Martin White, Christian Wagner, Salman Habib, David Higdon. Astrophys.J.715:104-121 (2010). arXiv:0812.1052

[18] e.g. U.Pen. ApJ, 504, 601 (1998); J. Peacock, et al. Nature 410, 169-173 (2001); Max Tegmark, Andrew J. S. Hamilton, Yongzhong Xu. MNRAS, 335, 887 (2002); M. Tegmark, et al. ApJ, 606, 702 (2004); Pengjie Zhang, Michele Liguori, Rachel Bean, Scott Dodelson. PRL, 99, 141302 (2007); Viviana Acquaviva, Amir Hajian, David N. Spergel, Sudeep Das. PRD, 78, 043514 (2008); Bhuvnesh Jain, Pengjie Zhang. PRD, 78, 063503 (2008); L. Guzzo, et al. Nature 451:541545,2008; Pengjie Zhang, Rachel Bean, Michele Liguori, Scott Dodelson. arXiv:0809.283 (2008); Eric V. Linder. Astropart.Phys.29:336 (2008); Yun Wang. JCAP, 0805,021(2008); Martin White, Yong-Seon Song, Will J. Percival. MNRAS, 397,1348 (2008); Reinabelle Reyes, Rachel Mandelbaum, Uros Seljak, Tobias Baldauf, James E. Gunn, Lucas Lombriser, Robert E. Smith. Nature, 464, 256-258 (2010)

[19] P.J.E. Peebles. ApJ, 205, 318 (1976); Limin Wang, Paul J. Steinhardt. ApJ, 508, 483 (1998); E.V. Linder. PRD, 72, 043529 (2005)

[20] Gravitational potential sources dark energy fluctuation through a term $(1+w) \dot{\phi}[7]$. Hence unless $w=-1$, dark energy fluctuation is inevitable. More rigorous proof can be achieved by resorting to the full linearized energy- momentum conservation equations in [7]. 